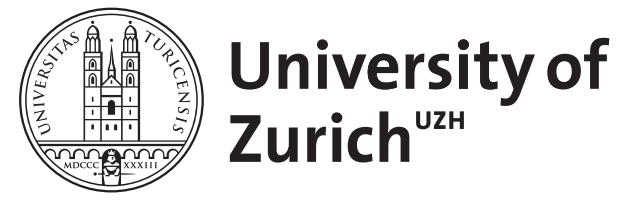

\title{
Die Eschatologie des Ereignisses. Zum messianischen Universalismus von Lost und Badiou
}

\author{
Boutin, Stéphane
}

Posted at the Zurich Open Repository and Archive, University of Zurich ZORA URL: https://doi.org/10.5167/uzh-171104

Book Section

Published Version

Originally published at:

Boutin, Stéphane (2015). Die Eschatologie des Ereignisses. Zum messianischen Universalismus von Lost und Badiou. In: Lörke, Tim; Walter-Jochum, Robert. Religion und Literatur im 20. und 21. Jahrhundert. Motive, Sprechweisen, Medien. Göttingen: VR unipress, 469-494. 
Sonderdruck aus

Tim Lörke / Robert Walter-Jochum (Hg.)

\section{Religion und Literatur im 20. und 21. Jahrhundert}

Motive, Sprechweisen, Medien

Mit 18 Abbildungen

V\&R unipress

ISBN 978-3-8471-0375-2

ISBN 978-3-8470-0375-5 (E-Book)

ISBN 978-3-7370-0375-9 (V\&R eLibrary) 



\section{Inhalt}

Tim Lörke und Robert Walter-Jochum

Religion und Literatur im 20. und 21. Jahrhundert. Zu diesem Band . . . 9

\section{Motive}

Hans Richard Brittnacher

Judas oder: Die Unvermeidlichkeit des Bösen. Literarische

Lösungsversuche eines theologischen Rätsels . . . . . . . . . . . . . . . 17

Mathias Meert

Patriarch und Prophet. Erwählung und Prüfung als religiöse Erfahrungen in Beer-Hofmanns Jaákobs Traum und Zweigs Jeremias ．.. . . . . . . 33

Yvonne Nilges

Thomas Mann und die Religion . . . . . . . . . . . . . . 51

Markus Schleich

»Even Jesus Wanted a Little More Time«. Die Passion Christi bei Tom

Waits, Nick Cave und Johnny Cash _ . . . . . . . . . . . . . . . 75

Franziska Thiel

„Der wahre Weltuntergang ist die Vernichtung des Geistes«. Die

Apokalypse als religiöse Form im Ersten Weltkrieg am Beispiel von Karl

Kraus' Die letzte Nacht und Kriegsbildern von Otto Dix . . . . . . . . . . 97

Imke Rösing

Die politische Instrumentalisierung der Apokalypse in der nationalsozialistischen Literatur am Beispiel von Joseph Goebbels' Roman Michael . . . . . . . . . . . . . . . . . . . . . . . . . . 119 
Markus Kraiger

Islamischer Fundamentalismus in der deutschen Gegenwartsliteratur.

Eine Analyse von Christoph Peters' Roman Ein Zimmer im Haus des

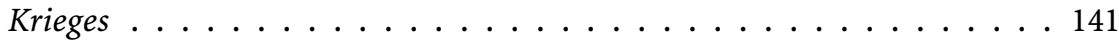

Frank Weiher

Wo ist mein Gott? Er hat mich verlassen! Über religiöse Motive des

Vampirismus in Bram Stoker's Dracula und True Blood . . . . . . . . . . 161

Laura Gemsemer

Matriarchale Freizügigkeit und mormonische Abstinenz. Religiöse

Elemente in P. C. und Kristin Casts House of Night Novels und in

Stephenie Meyers Twilight-Saga . . . . . . . . . . . . 181

Monika Wolting

Auswege aus der Eigenverantwortlichkeit? Religion, Esoterik und

Parapsychologie in Daniel Kehlmanns F . . . . . . . . . . . . 203

\section{Sprechweisen}

Wolfgang Braungart

Religion, Subjektivität, Autorschaft. Am Beispiel von Heines Tannhäuser

(1836) und Irmgard Keuns Gilgi - eine von uns (1931) . . . . . . . . . 227

Jens Ole Schneider

>Leben` als säkulare Ersatzreligion? Monistischer Weltdeutungsanspruch

und perspektivisches Sprechen in Hugo von Hofmannsthals Briefen des

Zurückgekehrten . . . . . . . . . . . . . . 255

Ariane Totzke

Schwindsüchtige Erlöser, psychotische Pfaffen und der »Fall Barbin«.

Oskar Panizzas ästhetischer Vandalismus im Deutschen Kaiserreich . . . 277

Tim Lörke

Ästhetisches Kerygma. Zur religiösen Dimension der Gedichte Gottfried

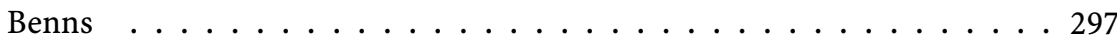

Lukas Pallitsch

Dichtung an der Schwelle: Zwischen dem Irrealis der Gegenwart und der

Erinnerung an die Zukunft . . . . . . . . . . . . . . 317 
Paweł Piszczatowski

Theologische Brocken in Gedichten von Paul Celan aus dem Band Die

Niemandsrose ........................ . . 335

Brigitte Schwens-Harrant

Literatur als Litanei . . . . . . . . . . . . . . . . . . . . . . 353

Robert Walter-Jochum

Vom Bekenntnis zum Plädoyer: Religion, Sexualität und Identität bei

Jean-Jacques Rousseau und Josef Winkler . . . . . . . . . . . . . . . 367

Alina Timofte

Das Gebet eines Hyperchristen? Zur Dekonstruktion einer religiösen

Gattung in Frost von Thomas Bernhard . . . . . . . . . . . . . . . . 391

Stefanie Burkhardt

Das Heilige und das Phantastische. Religionswissenschaftlicher

Exotismus in der indienbezogenen Literatur Mircea Eliades . . . . . . 405

Ludmila Peters

Postkoloniales Sprechen von Religion und Religiosität - Ilija Trojanows

Roman Der Weltensammler . . . . . . . . . . . . . . 423

Marie Gunreben

Literatur als Theodizee? Providenz und Kontingenz erzählter Welten am

Beispiel von Wolfgang Herrndorfs Sand . . . . . . . . . . . . . . 447

Stéphane Boutin

Die Eschatologie des Ereignisses. Zum messianischen Universalismus von Lost und Badiou . . . . . . . . . . . . . . . . . . . . . . . 469

Swen Schulte Eickholt

Sehnsucht nach der Immanenz. James Camerons Avatar - Aufbruch nach

Pandora als Utopie des göttlichen Menschen . . . . . . . . . . . . . 495

\section{Medien}

Peter Sprengel

Gladius Dei. Bild und Religion in Erzähltexten um 1900 . . . . . . . . 517 
Paul Onasch

»Wenn einer sich umgebracht habe, dürfe er nicht christlich begraben werden.« Kirchengeschichtliche Diskurse in den Romanen Uwe Johnsons

Michael Fisch

»Ich liebe den Tourismus. Er ersetzt die Völkerwanderung«. Hubert

Fichtes Blick auf Islam und Koran in dessen Erzählzyklus Die Geschichte

der Empfindlichkeit . . . . . . . . . . . . . . . . . 571

Ralph Olsen und Sebastian Kuppel

Das religionskritische Bilderbuch Wo bitte geht's zu Gott? fragte das

kleine Ferkel - Anmerkungen zur Rezeption . . . . . . . . . . . . . 591

Stefan Neuhaus

"Lasst Brian frei!« Religion und Postmoderne am Beispiel von Monty

Python's Life of Brian (GB 1979) . . . . . . . . . . . . . . . . . . . 609

Benjamin Beil

Göttliche Leerstellen - Religiöse Perspektiven des Computerspiels . . . . 625

Kai Matuszkiewicz

Religion in digitalen Spielen - Eine Frage des Genres . . . . . . . . . 645

Christian Dölker und Lorenz Trein

»But try to remember, this is a work of fiction." Zur Fiktionalisierung

von Religion in Assassin's Creed . . . . . . . . . . . . . . . . . . . 667

Robert Baumgartner

»Drücken Sie `Enter`, um Gott zu töten ...« - Das Computerspiel als

Medium des Deizids . . . . . . . . . . . . . . . 681

Autorinnen und Autoren . . . . . . . . . . . . . . 705 


\section{Die Eschatologie des Ereignisses. Zum messianischen Universalismus von Lost und Badiou}

Es gibt keine strikte, unverrückbare Trennlinie zwischen religiösem und säkularem Denken. Aber es gibt fortwährende Grenzstreitigkeiten, an denen man sich orientieren kann: Es gibt Expansionsbewegungen und Aneignungsversuche, Kompromisse und Verträge, es gibt den grenzüberschreitenden Handel mit Argumenten und die nicht deklarierte Zirkulation des Ideenschmuggels. Deshalb stellen sich die hier verhandelten Fragen jeder Gegenwart wieder von Neuem: Wie steht es heute um diese Grenze? Wo wird sie gerade verwischt, wo neu gezogen? Und nicht zuletzt: Was für Geschichten erzählt man sich aus dieser Grenzzone?

Eine paradigmatische Erzählung des beginnenden 21. Jahrhunderts, die sich auf geradezu obsessive Weise mit Fragen der Spiritualität beschäftigt, ist die populäre TV-Serie Lost. ${ }^{1}$ Als Produkt des unterhaltungsindustriellen Netzwerkfernsehens formal ein säkularer Text, wird die Serie inhaltlich jedoch so stark von religiösen Motiven geprägt, dass sie exakt in jener Grenzzone zwischen den beiden Bereichen spielt. Im Folgenden wird daher das für Lost zentrale Motiv der Erlösung als eine Scharnierstelle zwischen säkularem und religiösem Denken analysiert, wo die Logiken des Dies- und Jenseitigen ineinandergreifen und um die Deutungshoheit über das narrative Geschehen ringen. Wie sich zeigen wird, kann zur Klärung dieser Dynamik zudem die Ereignistheorie des atheistischen Philosophen Alain Badiou beigezogen werden. ${ }^{2}$ Zunächst jedoch sind in einem ersten Schritt einige der aporetischen Grundstrukturen herauszuarbeiten, denen sich die Beschäftigung mit Lost stellen muss.

1 Lost. Creators: J. J. Abrams, Jeffrey Lieber u. Damon Lindelof. USA: ABC 2004-2010 (im Folgenden direkt im Text zitiert nach Staffel und Episode, z. B. 1.1 für Staffel 1, Episode 1).

2 Vgl. Alain Badiou: Paulus. Die Begründung des Universalismus. Übers. v. Heinz Jatho. Zürich/Berlin 2009, 9. 


\section{Erzählspiel ohne Lösung? Die Aporien von Lost}

Wovon handelt Lost? Es gehört zu den Schwierigkeiten dieser Serie, dass sie selbst bei den einfachsten Fragen eine beträchtliche Vorsicht der Beantwortung fordert. Damon Lindelof, einer der Erfinder und Hauptautoren von Lost sowie als Showrunner zusammen mit Carlton Cuse verantwortlich für die ausführende Produktion, hat die Serie auf einer Pressekonferenz im Januar 2007, zur Hälfte der sechsjährigen Laufzeit, wie folgt zusammengefasst:

This show is about people who are metaphorically lost in their lives who get on an airplane and crash on an island and become physically lost on the planet Earth, and once they are able to metaphorically find themselves in their lives again they will be able to physically find themselves in the world again. ${ }^{3}$

So viel kann man daher als Konsens voraussetzen: Der Absturz von Flug Oceanic 815 verschlägt eine scheinbar heterogene Gruppe von Menschen - die jedoch dadurch verbunden sind, dass sie alle sprichwörtlich verlorene Seelen sind - auf eine einsame Insel irgendwo im Pazifik. Die grundlegende Problematik, welche die Handlung von der ersten bis zur letzten Minute bestimmt, ist deshalb die Frage nach der Rettung dieser Menschen.

Lindelofs Handlungs-Abstract verrät zudem bereits drei wesentliche Merkmale der Serie. Erstens vernäht Lost die Rettung aus der sogenannten metaphorischen Verlorenheit seiner Figuren direkt mit der Rettung aus ihrer physischen Verlorenheit. Die Charaktere werden also entweder ganz oder gar nicht gerettet: Tritt die Rettung ein, betrifft sie nicht nur einen Aspekt (metaphorisch oder physisch), sondern durchläuft das gesamte Dasein der Figuren (metaphorisch und physisch). Die Frage der Rettung übersteigt in Lost daher stets das Konkrete und wird stattdessen als Problem einer umfassenden Erlösung dieser gestrandeten Existenzen behandelt. Zweitens wird damit explizit, dass Erlösung im Lost-Kosmos ganz selbstverständlich als eine von Beginn an im Rahmen des narrativ Möglichen zirkulierende Option vorausgesetzt wird. Die Hoffnung auf Erlösung ist zwar in die Ungewissheit ihrer Erfüllung eingebettet, aber eben damit als zentraler Fluchtpunkt in die Perspektive des Narrativs integriert. Sie ist für Lost gewissermaßen immer schon im Spiel - im Gegensatz etwa zum gänzlich erlösungsfrei konstruierten Serien-Kosmos von The Wire. ${ }^{4}$ Drittens schließlich postuliert Lost einen klaren Vorrang dessen, was hier als metaphorisch bezeichnet wurde: Erlösung ist ein Ereignis, das sich nur in den innersten Schaltkreisen der menschlichen Seele vollziehen oder verfehlen kann. Physische

3 Zitiert nach Maureen Ryan: "Lost« producers talk about setting an end date and much more. Online unter: http://featuresblogs.chicagotribune.com/entertainment_tv/2007/01/lost_pro ducers_.html (31.07.2014).

4 The Wire. Creator: David Simon. USA: HBO 2002-2008. 
Rettung allein ist zwar möglich und wird in der vierten Staffel auch durchgespielt, als einige Überlebende - die sogenannten Oceanic Six - den Weg zurück in die Zivilisation finden. Der Ausgang dieses Zwischenspiels wird als Vorblende jedoch bereits in der letzten Episode der dritten Staffel vorweggenommen: Weil sie innerlich noch nicht bereit dazu sind, müssen die Oceanic Six auch ihre äußere Rettung sogleich wieder stornieren. Weil sie auf der metaphorischen Ebene immer noch verloren sind, müssen sie auch physisch erneut verloren gehen und noch einmal auf die Insel zurückkehren.

Diese Polarität von metaphorischer Innerlichkeit und physikalischer ÄuBerlichkeit wird in der Lost-Rezeption auch mit dem Begriffspaar character vs. mythology adressiert. Während die Showrunner die Serie stets als characterdriven bezeichnet haben, ${ }^{5}$ hat die Forschung hingegen darauf hingewiesen, dass die meisten Lost-Figuren zwar stetig mit sich hadern, dass sie aber in diesem Hadern nur wenig Entwicklung zeigen. Darin, so vermutet etwa Erika JohnsonLewis, könnte ein Grund für das primär auf die Mythologie der Insel fokussierte Interesse der Fans liegen: "when the audience never learns much about Jack beyond his obsessive need to fix things, they may begin to read the continual development of the island narrative as more important over and against the general lack of long term character development. ${ }^{6}$ Damit sind die zwei zentralen Orientierungen in der narrativen Dynamik der Serie skizziert, die beiden Grundprobleme, deren Gewichtung über die Zugehörigkeit zur Character- oder Mythology-Schule der Lost-Exegese entscheidet: Wer sind diese Menschen? Und: Was ist das für eine Insel? Wie sich zeigen wird, führen jedoch beide Orientierungen in die Aporie. Weder der Character-noch der Mythology-Ansatz können das Rätsel Lost lösen.

Wer also sind diese Menschen ${ }^{7}$ Auffallend ist die multikulturelle Zusammensetzung der Gestrandeten. Menschen verschiedener Nationen, Angehörige diverser Ethnien, Abgesandte aller Kontinente der Erde sind hier wie in einem globalen Querschnitt versammelt: die Passagiere eines Langstreckenflugs als heterogenes, wenn auch nicht repräsentatives Abbild der Menschheit zu Beginn des 21. Jahrhunderts. Man findet hier tätowierte Chirurgen (Jack Shephard), wundersam geheilte Paraplegiker (John Locke), irakische Folterknechte (Sayid Jarrah), flüchtige Kriminelle (Kate Austen), übergewichtige Lottogewinner

5 So sagte Cuse auf der bereits erwähnten Pressekonferenz: »We always view the show as a character show with a mythology frosting over the top«, zit. n. Ryan: „Lost« producers talk about setting an end date (Anm. 3).

6 Erika Johnson-Lewis: »We Have to Go Back«: Temporal and Spatial Narrative Strategies. In: Randy Laist (Hg.): Looking for Lost. Critical Essays on the Enigmatic Series. Jefferson/London 2011, 11-24, hier 14.

7 Für einen Überblick über die Hauptfiguren vgl. Dietmar Dath: Lost. Zürich/Berlin 2012, 13-37. 
(Hugo `Hurley` Reyes), Redneck-Trickbetrüger (James `Sawyer` Ford), alleinerziehende Afroamerikaner (Michael Dawson), hochschwangere Australierinnen (Claire Littleton), heroinsüchtige Rockstars (Charlie Pace), asthmatische ItGirls (Shannon Rutherford), nigerianische Drogenhändler/Priester (Mr. Eko) und koreanische Paare mit Eheproblemen (Jin und Sun Kwon). Hinzu kommen entführte Fertilitätsforscherinnen (Juliet Burke), unsterblich verliebte schottische Ex-Mönche (Desmond Hume) und manipulative Massenmörder (Ben Linus).

Auch wenn die zentralen Erzählstränge dann doch meist anhand von weißen, englischsprachigen, heterosexuellen Männern entwickelt werden, ${ }^{8}$ suggeriert die Diversität des Ensembles dennoch eine parabelhafte Universalität dieser kollektiven Robinsonade. Was die Kollektivität überhaupt erst herstellt, ist darum nicht so sehr die Bedrohung durch das Fremde, durch die sogenannten Others, mit denen sich die Gestrandeten bald herumschlagen müssen. ${ }^{9}$ Das gemeinsamkeitsstiftende Merkmal dieser zusammengewürfelten Gemeinschaft von Überlebenden ist vielmehr genau die Universalität der Verlorenheit: Wenn Menschen von überall auf der Welt auf dieselbe Weise verloren gehen können, dann gehört lostness offenbar zur conditio humana - ähnlich wie gemäß Heidegger das Dasein immer schon im "Charakter des Verlorenseins« ans Seiende verfallen ist. ${ }^{10} "$ Last week most of us were strangers «, deklamiert Jack in einer ikonischen Ansprache an die sich formende Gemeinschaft: "But we're all here now. And God knows how long we're going to be here. But if we can't live together, we're going to die alone.« (1.5) Die Implikationen dieser Rede werden im Lauf der Zeit immer stärker als das eigentliche Mantra der Serie hervortreten: Wenn wir uns nicht gegenseitig finden können, werden wir an unserer je eigenen Verlorenheit zugrunde gehen.

Man hat Lost deshalb als paradigmatischen "post-9/11-text" gelesen, als Erzählung über das Trauma des Westens in Zeiten der Globalisierung, als moderne Odyssee einer entwurzelten Mobilität: "the unhomely has become a constant state of being and 'going homer is not as simple as it used to be«, wie Aris Mousoutzanis schreibt. ${ }^{11}$ Doch auch wenn Lost diese nostalgischen Klangfarben

8 Vgl. dazu David Magill: The Lost Boys and Masculinity Found. In: Laist (Hg.): Looking for Lost (Anm. 6), 137-153.

9 Diese These vertritt Peter S. Fosl: Friends and Enemies in the State of Nature: The Absence of Hobbes and the Presence of Schmitt. In: Sharon Kaye (Hg.): The Ultimate Lost and Philosophy. Think Together, Die Alone. Hoboken 2011, 164-186. Dagegen argumentiert Karen Gaffney, dass Lost das Konzept von Otherness gerade dekonstruiere, vgl. dies.: Ideology and Otherness in Lost: »Stuck in a Bloody Snow Globe«. In: ebd., 187-206.

10 Martin Heidegger: Sein und Zeit. Tübingen 2001 [1927], $175 \mathrm{ff}$.

11 Aris Mousoutzanis: „Enslaved by Time and Space«: Determinism, Traumatic Temporality, and Global Interconnectedness. In: Laist (Hg.): Looking for Lost (Anm. 6), 43-58, hier 52, 55. Vgl. dazu auch Jesse Kavadlo: We Have to Go Back: Lost After 9/11. In: ebd., 230-242. 
durchaus anklingen lässt, ist das zentrale Thema der Serie auf anderen, abstrakteren Akkorden aufgebaut. Denn die Traumata der Gestrandeten betreffen nicht so sehr den Flugzeugabsturz, den sie gerade erlebt haben. Wie das $\mathrm{Pu}-$ blikum in unzähligen Rückblenden in die Vergangenheit der Figuren erfährt, reichen die Wurzeln ihrer Verlorenheit sehr viel tiefer. Die Rückblenden erzählen im Wesentlichen immer wieder dieselben zwei Motive: einerseits ein fortwährendes Ringen der Figuren mit einer im Allgemeinen familiär triangulierten Situation der Schuld oder der Verlassenheit. ${ }^{12}$ Und andererseits eine Reihe von Kreuzungspunkten, an denen die Verlorenen sich bereits präinsular scheinbar zufällig begegnen oder Verbindungen zwischen ihnen aufleuchten.

Damit erzielt Lost zwei Effekte: Erstens werden alle Figuren nach den immer selben Parametern als verloren und dementsprechend erlösungsbedürftig gezeichnet. Dieses Muster wird dermaßen stereotyp durchexerziert, dass Lindelof im Audiokommentar zu einer Szene, als Sawyer wieder einmal mit nacktem Oberkörper auftritt, sogar selbstironisch über eine mögliche Rückblende aus der Sicht des verlorenen Hemds scherzen kann: »the manufacturing mill where it's made, and then how the shirt ended up in Sydney and got on Oceanic 815, and what the shirt is trying to redeem itself for.« (4.14) Andererseits ergibt sich durch die allzu zufälligen Begegnungen der unabweisbare Eindruck, dass hinter diesem Flugzeugabsturz und der daraus entstehenden Gemeinschaft der Verlorenen mehr steckt als eine bloße Verkettung von unglücklichen Umständen. »Diese Leute«, resümiert Dietmar Dath, »müssen durch etwas hindurch, das sich in den Nöten der Immanenz, im Stress des Diesseitigen nicht erschöpft «. ${ }^{13}$

Die Frage ist nur: durch was genau? In einem Interview vom Mai 2010, kurz vor der Ausstrahlung der letzten Lost-Folge, antwortet Lindelof auf die Frage nach den wichtigsten Themen der Serie mit den beiden Begriffen der Erlösung und der Gemeinschaft:

If there's one word that we keep coming back to, it's redemption. [...] But in order to redeem yourself, you can only do it through a community. [... T] hese people were all lone wolves who were complete strangers on an aircraft $[\ldots]$. Then let's bring them together and through their experiences together allow themselves to be redeemed. ${ }^{14}$

Die Hauptthese der Character-Schule lautet dementsprechend, dass das Engagement in der Gruppe oder das Opfer für die Gemeinschaft die betreffende Figur

12 Vgl. Holly Hassel u. Nancy L. Chick: »It Always Ends the Same«: Paternal Failures. In: Laist (Hg.): Looking for Lost (Anm. 6), 154-170, hier 154:»Whether absent, abusive, or doomed, the show's fathers force the main characters to grapple with their own father's failures or their own failures as fathers".

13 Dath: Lost (Anm. 7), 18.

14 Zitiert nach Lorne Manly: The Men Who Made ABC’s "Lost« Last. Online unter: www. nytimes.com/2010/05/16/arts/television/16weblost.html (31.07.2014). 
erlöse. »In the end «, meint etwa Sarah Clarke Stuart, »everyone who cooperated and sacrificed themselves for the group was redeemed. ${ }^{15}$ Ebenso behauptet Brett Chandler Patterson: "Once characters have faced their past failures, [...] they may find redemption, but this opportunity for a new start, for a second chance, arrives primarily in their becoming a part of the community of survivors. ${ }^{16}$

Doch damit macht man es sich zu einfach. Manche Figuren bleiben unerlöst, obwohl sie der Gemeinschaft gute Dienste leisten oder sogar ihr Leben für sie aufgeben. Michael etwa opfert sich, als letzter im Bauch des Frachters zurückbleibend, um die Explosion der Bombe so lange wie möglich hinauszuzögern und den anderen so die Gelegenheit zur Flucht zu geben (4.14). Trotzdem ist er offenbar nicht erlösungswürdig und muss als Geist mit anderen Nichterlösbaren weiter auf der Insel ausharren, wie er Hurley erklärt: »We're the ones who can't move on.« (6.12) Desgleichen entwickelt sogar Ex-Bösewicht Ben gegen Ende der Serie eine bemerkenswerte Teamfähigkeit im Dienst des Guten (6.7, 6.16, 6.18, ebenso im Epilog The New Man in Charge). Trotzdem scheint ihm noch etwas Wesentliches zu fehlen: "I have some things I still need to work out." (6.18) Erlösung ist also eine komplizierte Prozedur, sie folgt nicht automatisch aus jenen simplen Regeln, welche der Character-Ansatz postuliert.

Der Grund dafür wurde bereits genannt: Die Immanenz ist nie genug. Das Schicksal dieser Figuren erschöpft sich nicht im Diesseitigen. Wie Cuse im erwähnten Interview zu Protokoll gibt, erzählt Lost vor allem eine spirituelle Reise - »a spiritual journey «. ${ }^{17}$ Daraus folgt, dass die Geschichte letztlich nicht allein aus der situationsimmanenten Dynamik menschlicher Beziehungen und Charakterentwicklungen verstanden werden kann. Um Lost aufzuschlüsseln, wird man also auf die spirituelle Ebene der Mythologie zurückgreifen müssen. Vom Character-Ansatz zur Mythology-Schule der Inselkunde hinüberwechselnd, begegnet einem als Erstes die Frage, wie diese Figuren überhaupt auf der Insel gelandet sind. Dazu bietet Lost zwei Erklärungen an: Einerseits zeigt die Episode 2.24, wie Desmond exakt am Tag des Absturzes von Oceanic 815 in der Swan Station einen elektromagnetischen Unfall auslöst, der das Flugzeug vom Himmel geholt haben könnte. Was es mit den vorgängigen schicksalshaften Begegnungen auf sich hat, wird damit jedoch nicht geklärt.

Andererseits ist aus den Episoden 5.16/17, 6.9 und 6.15 bekannt, dass der erst gegen Serienende enthüllte Beschützer der Insel, Jacob, und sein Bruder, der namenlose Man in Black, in eine womöglich seit Jahrtausenden laufende Wette

15 Sarah Clarke Stuart: Literary Lost. Viewing Television Through the Lens of Literature. New York 2011, 143.

16 Brett Chandler Patterson: The New Narnia: Myth and Redemption on the Island of Second Chances. In: Kaye (Hg.): The Ultimate Lost and Philosophy (Anm. 9), 253-279, hier 276.

17 Zitiert nach Manly: The Men Who Made ABC’s »Lost« Last (Anm. 14). 
über die fundamentale Güte oder Boshaftigkeit des Menschengeschlechts verstrickt sind. ${ }^{18}$ Dazu holt Jacob immer wieder neue Menschen auf die Insel, um endlich ihre Güte zu beweisen, während sein Bruder - bisher offenbar stets mit Erfolg - versucht, sie zu korrumpieren. Wie Jacob dabei genau vorgeht, bleibt jedoch unklar. Ist er eine allmächtige Gottheit, welche die Menschen nur einmal kurz zu berühren braucht, um ihren Pfad auf Jahrzehnte hinaus zu bestimmen, wie in den Rückblenden der Episoden 5.16/17 suggeriert wird? Oder ist er einfach nur ein geschickter Manipulator, wie die Äußerungen des Man in Black gegenüber Sawyer vermuten lassen?

At some point in your life, James, probably when you were young, when you were miserable and vulnerable, he came to you. He manipulated you, pulled your strings like you were a puppet. And, as a result, choices that you thought you made were never really choices at all. He was pushing you, James. Pushing you - to the island. (6.4)

Die Serie ist sich in diesem Punkt uneins. Einerseits wird Jacob deutlich als ein Wesen mit übernatürlichen Fähigkeiten gekennzeichnet, etwa wenn er seinem Gehilfen Richard Alpert ewiges Leben verleiht (6.9). Andererseits präsentiert Lost zum Hintergrund der Figur Jacob kaum Mythologie, sondern im Gegenteil vor allem Character-Erklärungen.

In der dafür zentralen Episode 6.15 wird die Vorgeschichte von Jacob und seinem Bruder als ein weiteres Drama in jener Logik manipulativer oder abwesender Elternschaft erzählt, welche bereits die meisten Rückblenden in die Vergangenheit der Gestrandeten strukturierte. Diese Genealogie markiert Jacob als Menschen, nicht als Gott. Sie zeigt ihn als verlorenen und verletzten Erdensohn, adoptiert und aufgezogen von jener namenlosen Frau, die seine Mutter ermordet hat, um in ihm über einen Nachfolger für ihre Position als Inselbeschützerin zu verfügen. Ebenso wird der Man in Black von derselben falschen Mother am Verlassen der Insel gehindert, zum Mord an ihr verführt und daraufhin durch Jacobs Rache versehentlich zu einem rauchförmigen Symbol des Bösen potenziert. Die Episode erzählt damit zwar die Herkunft der übernatürlichen Kräfte des Man in Black, diejenigen Jacobs bleiben jedoch bloße Behauptung. So unterstreicht auch Lindelof im Audiokommentar zu dieser Folge noch einmal, dass die Serie sich in der Tat mehr für "manipulative parents" als für das Übernatürliche interessiere: "Lost is really a study in bad parenting." (6.15)

Dass dieselbe Episode 6.15 dann trotzdem noch eine Höhle mit einem esoterisch leuchtenden Licht des Lebens aufbietet, um die Mythologie der Serie nicht ganz in der Luft hängen zu lassen, wirkt deshalb eher sekundär. Die in der

18 Einer der wichtigsten Referenztexte von Lost ist die Bibel, hier etwa die Wette zwischen Gott und Satan im Buch Hiob. Vgl. dazu Stuart: Literary Lost (Anm. 15), insbesondere $17 \mathrm{f}$. und 54-88. 
letzten Staffel eingeführten sogenannten Seitwärtsblenden (flash sideways) dienen nicht zuletzt dem Zweck, den auf der Insel tobenden Endkampf zwischen Gut und Böse - den Kampf um die Bewahrung oder die Zerstörung jenes Lichts beträchtlich zu relativieren. Die Handlung und das Format der jenseits der Insel spielenden Seitwärtsblenden perspektivieren diese mythischen Abenteuer zuletzt als mehr oder weniger nebensächlich. Die wirkliche Erlösung, so zeigen die Seitwärtsblenden, findet nicht dort statt, im Kampf mit Fäusten, Messern und Gewehren, sondern hier: in einem Geschehen, das vor allem in den Augen und den Gesichtern der Menschen spielt. ${ }^{19}$ Das Problem ist bloß, dass die Seitwärtsblenden - noch während sie das Inselgeschehen als peripher ausweisen gleichzeitig doch darauf bestehen, dass die finale Erlösung nicht ohne eine Affirmation eben jener Inselereignisse einsetzen kann. Jack stellt sich hierbei am schwierigsten an und weist die Affirmation bis zu den allerletzten Minuten der Serie beharrlich zurück. Darüber hinaus verdeutlichen die Seitwärtsblenden erneut, dass Erlösung nichts mit der Arbeit der Immanenz zu tun hat: Es reicht nicht aus, dass Jack in der Seitwärtswelt mit seinem Sohn David nicht dieselben Fehler macht wie sein Vater mit ihm (6.5) - daraus folgt noch keine Erlösung. Es braucht offensichtlich mehr, als was auf der Ebene von Charakterentwicklung und Beziehungspflege möglich ist, um vollends errettet zu werden. Es braucht eben doch eine Ergänzung, ein Supplement der Mythologie.

So geht das bei Lost bis in die mikroskopischsten Erklärungsverzweigungen: character verweist auf mythology, die Mythologie verweist zurück auf die Figuren. Antworten werden prinzipiell aufgeschoben und an die nächste $\mathrm{Zu}$ ständigkeitsstelle weiterverwiesen. Jede dieser Lektürevarianten endet früher oder später aporetisch:»Welcome to the wonderful world of not knowing what the hell's going on «, wie Kate sagt (3.15). Oder in den Worten Daths: »die Würfel fallen immer, bleiben aber nie länger liegen, als bis wir die neuen Zahlen abgelesen haben ${ }^{20}{ }^{20}$ Das Puzzle von Lost scheint somit unvollständig zu sein. Oder lassen sich die fehlenden Teile ergänzen? Grundsätzlich hat man hier drei Möglichkeiten zur Auswahl.

Erstens besteht in Lost immer die Option, dass die fehlenden Teile im Sinn eines leap of faith $^{21}$ schlicht übersprungen werden müssen: Man soll vielleicht einfach glauben und darauf vertrauen, dass die Serie schon weiß, was sie tut. So wie in der Gemeinschaft der Verlorenen immer wieder die Vertrauensfrage gestellt wird - »You just have to trust me« oder »I wish you believed me«, sagen die Figuren dann jeweils (vgl. etwa 6.14) -, kann man das Ende auch als Vertrau-

19 Vgl. dazu Dath: Lost (Anm. 7), 46.

20 Dath: Lost (Anm. 7), 30.

21 Nicht zufällig wird in Episode 6.2 unter dem Tempel eine französische Ausgabe von Kierkegaards Furcht und Zittern gefunden. Verwendet wird der Ausdruck leap of faith zum Beispiel in den Folgen 2.3, 5.6 und 6.13. 
ensfrage der Serie als Ganzes verstehen: This is the end, you just have to trust us. Das Motiv des Glaubenssprungs beschäftigt zudem bereits die ganze zweite Staffel. Darin treffen die Gestrandeten in der Swan Station auf Desmond, welcher die letzten drei Jahre damit verbracht hat, alle 108 Minuten manuell eine bestimmte Zahlenfolge in seinen Computer einzugeben, um damit - wie ihm gesagt wurde - das Ende der Welt immer wieder aufzuschieben. Es gehört zu den Spezialitäten von Lost, solche technisierten Analogien des Glaubens von allen Seiten her zu erforschen. Handelt es sich um einen Wink des Schicksals, wie Desmond zu glauben scheint? Ein verhaltenspsychologisches Experiment, wie Jack und nach der Entdeckung der Pearl Station auch Locke vermutet? Einen Test des Glaubens, wie Eko spekuliert? Die Auflösung verharrt in der für Lost üblichen Unentschiedenheit: Als Locke am Ende der zweiten Staffel die Eingabe verhindert, tritt tatsächlich eine Katastrophe ein, die jedoch durch Desmonds Fail-Safe-Schlüssel auf ein lokales Maß begrenzt werden kann.

Zweitens kann der Narration von einer Metaebene aus Stabilität verliehen werden. Man hat zum Beispiel immer wieder bemerkt, wie sehr die Architektur von Lost an die aus Videogames bekannten Schemata der Rätsel- und Rollenspiele erinnert: Die verschiedenen Figuren offerieren dem Publikum gewisse Rollen, in denen man die Serie durchspielen, aber auch sterben kann. Als Belohnung für die Erfüllung bestimmter Aufgaben werden neue Spielorte freigeschaltet sowie Waffen, Nahrung oder sonstige Rettungspunkte gesammelt. Außerdem gibt es einen Schwierigkeitsfortschritt nach Levels, auf denen die Aufgaben, Gegner und Spielorte jeweils neu definiert werden. ${ }^{22}$ Daran knüpft Dath an, wenn er Lost als Erzählspiel ${ }^{23}$ betrachtet, in dem »Spiel und Widerspiel [...] nichts anderes sind als die Formen der gebundenen und der freien Phantastik selbst «: Immanente Ausweglosigkeit spielt gegen transzendenten Ausweg. Das letzte Level - der Ausweg aus dem Spiel - bestünde dann darin, diesbezüglich auf endgültige Antworten verzichten zu können: »Was diese Geschichte nicht zum Wenigsten will, ist, den Leuten bestimmte Antwortbegehren auszutreiben; sie verlangt, dass Rätsel nicht geknackt, sondern für bessere aufgegeben werden müssen ${ }^{24}$

22 Vgl. dazu die anonyme Website: Lost is a Game. Online unter: lostisagame.com (31.07. 2014), ebenso Steven E. Jones: The Game of Lost. In: ders.: The Meaning of Video Games. Gaming and Textual Strategies. New York 2008, 19-46, und Jennifer Buckendorff: Fans play TV series »Lost like an interactive video game. Online unter: http://seattletimes.com/html/ entertainment/2002730079_lostgame10.html (31.07.2014). Lost ist außerdem paradigmatisch für die neuen Weisen der interaktiven fan participation, vgl. Sandra Ziegenhagen: Zuschauer-Engagement. Die neue Währung der Fernsehindustrie am Beispiel der Serie "Lost $«$. Konstanz 2009.

23 Zum Begriff des Erzählspiels vgl. Albrecht Koschorke: Wahrheit und Erfindung. Grundzüge einer Allgemeinen Erzähltheorie. Frankfurt a. M. 2013, 12.

24 Dath: Lost (Anm. ), 62, 49. 
Eine dritte Option schließlich besteht darin, ein externes Erklärungsmuster hinzuzuziehen, ohne die Auslegung deshalb gleich auf eine Metaebene zu verlegen. Im Folgenden wird dieser Weg eingeschlagen, indem Lost anhand von Badious Ereignistheorie aufgeschlüsselt wird.

\section{Jenseits der Immanenz: Badiou und die Gnade des Ereignisses}

Die gemeinsame Grundthese von Lost und Badiou besteht in der Behauptung, dass es so etwas wie eine universale Singularität geben soll. ${ }^{25}$ Die Form, in der eine solche Entität auftrete, nennt Badiou ein Ereignis. Wie er in seinem Hauptwerk Das Sein und das Ereignis schreibt, versucht er damit aufzuzeigen, »wie das Sein ergänzt werden kann«. Man hat es hier also grundsätzlich mit einem Denken zu tun, dem das Sein nicht genügt: Diese Philosophie sucht nach etwas, das mehr ist als das Sein, anders als das Sein, weil Sein für Badiou immer nur »das geregelte Spiel der Vielheit« umfasst und sich bloß in der Ordnung der jeweiligen Situation, als ein »in-Situation-zu-sein « präsentiert. ${ }^{26}$ Badious Denken operiert stets in dieser Doppelung: Es praktiziert eine Abwertung des Seins als ereignislosem Zustand einer starren Strukturiertheit durch die Regeln der Normalität - und evoziert gleichzeitig die Hoffnung auf einen Einbruch des absolut Anderen, auf eine Öffnung der Situation hin zu einer kommenden Wahrheit. Der Dualismus Badious verfügt daher eine fundamentale Spaltung der Entitäten: auf der einen Seite das Sein (Determination, Struktur, Normalität) und auf der anderen Seite das Ereignis (Gnade, Unterbrechung, Wahrheit). ${ }^{27}$

Auch wenn Badiou keinen prophetischen oder teleologischen Messianismus vertritt, zeigt seine Ereignistheorie dennoch eine gewisse messianische Qualität: Sie verspricht die Möglichkeit einer weltverwandelnden Errettung aus der erlebnisarmen Repetition des Gewöhnlichen. Es gibt, so lässt der Begriff des Ereignisses hoffen, etwas jenseits der schalen Ordnung des Alltags, in der stets nur das geschieht, was die Berechenbarkeit des Endlichen nicht überschreitet. Das

25 Badiou: Paulus (Anm. 2), 21: „Welches sind die Bedingungen einer universalen Singularität?« Für wertvolle Hinweise zu Badiou und die kritische Diskussion der folgenden BadiouInterpretation danke ich Karin Schraner.

26 Alain Badiou: Das Sein und das Ereignis. Übers. v. Gernot Kamecke. Berlin 2005, 31, 45, 52.

27 Zu Badious Dualismus vgl. etwa Slavoj Žižek: From Purification to Subtraction: Badiou and the Real. In: Peter Hallward (Hg.): Think Again. Alain Badiou and the Future of Philosophy. London/New York 2007, 165-181, hier 174ff., ebenso Simon Critchley: Unendlich fordernd. Ethik der Verpflichtung, Politik des Widerstands. Übers. v. Andrea Stumpf u. Gabriele Werbeck. Zürich/Berlin 2008, 56ff., sowie Oliver Marchart: Die politische Differenz. Zum Denken des Politischen bei Nancy, Lefort, Badiou, Laclau und Agamben. Berlin 2010, 177. 
Pathos des Ereignisses setzt sich von all dem ab und zieht den Zauberkreis der besonderen Seltenheit um sich: Es gibt Außerordentliches und Wundersames, es gibt Unendliches in der Welt. Im zweiten Band seines Hauptwerks wird Badiou das Ereignis deshalb durch ein »Maximalwerden der Existenzintensität« kennzeichnen. ${ }^{28}$ Dass Badiou nun aber zu jenen vier Prozeduren, in denen ein solches Plus an Existenzintensität realisiert werden kann, neben den eher klassischen Kandidaten der Kunst, der Wissenschaft und der Politik auch die romantische Liebe zählt, lässt, wie Slavoj Žižek bemerkt hat, diese etwas aus der Rolle fallende vierte Prozedur als formales Raster der übrigen drei erscheinen. ${ }^{29}$

Am Beispiel der Liebe kann man folglich besonders gut beobachten, wie sich das Außerordentliche vollzieht: wie sich "aufgrund des bloßen Zufalls einer Begegnung « ein absoluter Schnitt ereignet, der »vom Punkt des Liebes-Ereignisses" aus das Besondere festhält und »die Folgewirkungen der Liebe vom gewöhnlichen Lauf der Dinge unterscheidet«. Und wie sich in dieser Befreiung von der Totalität des Gewöhnlichen jene neue Gesamtheit des Erlebens konstituiert, welche "jenseits der bloßen Begebenheit die Welt der Liebe ausmacht «. ${ }^{30}$ Was bisher unter dem Begriff Ereignis zusammengefasst wurde, erweist sich bei genauerem Hinsehen daher als wechselseitiges Zusammenwirken dreier Komponenten. In Bezug auf ein Liebesereignis unterscheidet Badiou erstens das »Wunder der Begegnung«, zweitens »das mühsame Werden einer Punkt für Punkt konstruierten Wahrheit« und drittens »das Glück als immanente Belohnung der Mühe «. ${ }^{31}$ Trotzdem sind diese drei Komponenten derart aufeinander bezogen, dass sie nicht isoliert betrachtet werden können.

Das eigentliche Ereignis besteht in der "Überraschung" einer Begegnung, welche "nach den Gesetzen der Welt weder vorhersehbar noch berechenbar war«. Gerade deshalb ist das Ereignis aber auch äußerst flüchtig. Wird es nicht als Ereignis benannt und als Ausgangspunkt einer Wahrheitskonstruktion festgehalten, dreht sich die Welt einfach weiter und alles wird genauso sein, als ob es nie stattgefunden hätte. Darum kann man in einem gewissen Sinn sagen, dass sich das Ereignis (erste Komponente) tatsächlich erst dann ereignet hat, wenn man die Entscheidung fällt, den »Zufall der Begegnung" nicht einfach vergehen zu lassen, sondern ihn »in der Form eines Anfangs zu fixieren « (zweite Komponente). Die Liebeserklärung ist ein solcher Eingriff, der festhält: »Ich werde aus dem, was ein Zufall war, etwas anderes machen. Ich werde daraus eine Dauer, eine Hartnäckigkeit, eine Verpflichtung, eine Treue machen.« Das Subjekt

28 Alain Badiou: Logiken der Welten. Das Sein und das Ereignis 2. Übers. v. Heinz Jatho. Zürich/ Berlin 2010, 609.

29 Žižek: From Purification to Subtraction (Anm. 27), 170.

30 Badiou: Das Sein und das Ereignis (Anm. 26), 263.

31 Alain Badiou: Lob der Liebe. Ein Gespräch mit Nicolas Truong. Übers. v. Richard Steurer. Wien 2011, 67. 
allerdings, das diese Entscheidung fällt, ist für Badiou nicht bereits gegeben, sondern konstituiert sich überhaupt erst in einer solchen Entscheidung, in der Treue zu einem Ereignis (dritte Komponente): »Denn sie und ich, wir sind Teil dieses einzigen Subjekts, dieses Liebessubjekts, das die Entfaltung der Welt durch das Prisma unseres Unterschieds sieht, sodass die Welt sich ereignet und geboren wird «. ${ }^{32}$

Badious Theorie zeichnet sich nun gerade dadurch aus, dass sie ihren Messianismus auf die gesamte Dreifaltigkeit des Ereignisses verteilt. Sie lässt die Erlösung zwischen den drei Komponenten zirkulieren, ohne eine davon direkt eschatologisch zu besetzen. Denn für sich allein genommen ist keine Komponente unmittelbar mit einem Heilsversprechen verknüpft: Das Ereignis ist zwar als unberechenbares Vorkommnis durchaus außerordentlich, an sich kann es aber nichts und niemanden erlösen, sondern verpufft einfach in der Kontingenz. Die Treue generiert im Anschluss an das Ereignis zwar eine Wahrheit, an sich ist sie allerdings nicht durch Erlösung, sondern eher durch Arbeit, Mühe und Verpflichtung bestimmt. Das Subjekt schließlich bildet zwar den durch das Ereignis veränderten Modus des Welterlebens, an sich ist es jedoch nie präsent, es aktualisiert sich vielmehr immer nur performativ, im Vollzug der Treue. Und doch wird im Zusammenspiel dieser drei Komponenten eine Erwartungshaltung aufgebaut, die man nur als messianisch bezeichnen kann.

Die Funktionsweise dieses Zusammenspiels ist nicht ganz einfach zu verstehen. Sie hat jedoch direkt mit jener These einer universalen Singularität zu tun, welche auch die narrative Konstruktion von Lost bestimmt. Badiou lässt keinen Zweifel daran, dass er mit der Spaltung von Sein und Ereignis »die Existenz von zwei inkommensurablen Formen des In-Seins" postuliert. Trotzdem hält er daran fest, dass dieser Schnitt nur eine "Spaltung der Immanenz" darstelle: Sein und Ereignis wären gemäß Badiou als gleichermaßen immanent zu denken. ${ }^{33}$ In Das Sein und das Ereignis ist diese Schwierigkeit von Anfang an präsent: Wenn das Sein nur als strukturierte Vielheit präsentiert wird, so wird diese Struktur dennoch nicht einfach als gegeben, sondern als Resultat einer "Zählung-als-Eins« aufgefasst. »Damit das Eins jedoch resultiert", so das zentrale Argument, "muss es retwas in der Vielheit geben, das nicht absolut mit dem Resultat zusammenfällt«. Natürlich beeilt sich Badiou festzuhalten, dass tatsächlich »alles gezählt wird «, dass es keine »Lücke« im Sein gibt und dass man daher garantiert »keiner vereinzelten Insel begegnet», welche in der Zählung

32 Badiou: Lob der Liebe (Anm. 31), $32 \mathrm{ff} ., 42 \mathrm{f} ., 29$.

33 Alain Badiou: Afterword: Some Replies to a Demanding Friend. In: Hallward (Hg.): Think Again (Anm. 27), 232-237, hier 235: "scission of immanence (the existence of two incommensurable forms of being-in)« (Übersetzung im Text: S. B.). 
irgendwie »/vergessen«" worden wäre. Trotzdem hinterlasse die Zählung einen "phantomhaften Rest «. ${ }^{34}$

Doch wenn in der Tat alles gezählt wird, welcher geheimnisvolle Rest soll dann noch übrig bleiben? Wenn wirklich die gesamte Immanenz in die Zählung einfließt, kann das Resultat nur insofern von seinem Ausgangspunkt abweichen, als an irgendeinem Punkt eben doch eine wilde Transzendenz hineinfunkt und die Rechnung durcheinanderbringt. Badiou löst das Problem, indem er über der Ebene der Struktur (also der Situation) noch eine der Metastruktur (die sogenannte Verfassung der Situation) einzieht und damit die Rechnung verdoppelt was natürlich die Möglichkeit eines Rechenfehlers entsprechend vergrößert. Wo die Rechnung auf beiden Ebenen übereinstimmt, herrscht die Normalität. Wo die Metastruktur etwas erfasst, das die Struktur nicht zählt, hat man es mit einem Auswuchs zu tun. Und wo die Struktur etwas präsentiert, das von der Metastruktur nicht repräsentiert wird, liegt gemäß Badiou etwas Besonderes vor. Eine solche Singularität gehört zwar zur Situation, wird von ihr aber "nicht eingeschlossen«. Sie wird zwar gezählt, setzt sich jedoch aus Elementen zusammen, »die von der Zählung nicht erfasst werden«. Man ahnt bereits, dass nur an solchen Stellen, wo das Sein sich verrechnet, ein Ereignis einschlagen kann. Badiou nennt daher einen Ort, der »absolut besonders « ist, eine »Ereignisstätte«. Solche Stätten befinden sich "am Rand der Leere« - dort, wo das Außerordentliche beginnt. ${ }^{35}$

Lässt sich mit diesem Vokabular nicht auch das Setting von Lost beschreiben? Die Realität der Insel wird von der Serie immer wieder affirmiert: Sie ist keine Lücke im Sein, kein jenseitiges Fegefeuer und nicht auf die Verzerrungseffekte einer verwirrten Psyche reduzierbar. ${ }^{36}$ Sie zählt zum Sein und trotzdem existiert sie offiziell nicht, sie wird auf keiner Seekarte verzeichnet, die Metastruktur weiß nichts von ihr. Zugleich wimmelt es auf ihr von Elementen, welche innerhalb der situativen Ordnung nicht gezählt werden können: Eisbären in den Tropen? Eine bösartige Rauchsäule? Das intensive Flirren einer nicht abweisbaren Schicksalhaftigkeit? Die Insel wird damit als ein Ort des Besonderen gekennzeichnet, sie ist eine absolute Singularität, situiert am Rand der Leere eines unendlichen Ozeans, wo man verloren gehen, aber auch gefunden werden kann: Die Insel bildet eine Ereignisstätte.

Lost zielt jedoch immer schon über diese Singularität hinaus, in Richtung auf das größere Ganze eines Vorgangs, dessen Geheimnis sich nie auf das Partikulare einer einzelnen Figur reduzieren lässt. Wie Dath bemerkt: »Wir sollen glauben, dass diese Leute einander wichtig sind, [...] wobei das konkrete Ein-

34 Badiou: Das Sein und das Ereignis (Anm. 26), $69 \mathrm{ff}$.

35 Badiou: Das Sein und das Ereignis (Anm. 26), 119, 540.

36 Vgl. dazu Dath: Lost (Anm. 7), 85. 
zelschicksal sich in einem Zusammenhang auflösen darf, der keiner und keinem Einzelnen ganz gehört, von niemandem allein erzähl- oder erklärbar ist. ${ }^{37}$ Sowohl Lost als auch Badiou nehmen hier eine messianische Operation der Ergänzung vor: Beide markieren das Singuläre einer nicht in der gesetzten Ordnung aufgehenden Stätte als Einsatzpunkt einer jede Partikularität überschreitenden, universalen Ereignishaftigkeit. Doch wie kommt diese Universalität ins Spiel? Was genau ereignet sich in einem Ereignis?

Letztlich muss Badiou zugeben, dass seine Theorie dem »Wunder« des Ereignisses tatsächlich eine Art "metaphysischen Status« verleiht. ${ }^{38}$ In einer bemerkenswerten Wendung gesteht Badiou seine Bereitschaft, die Immanenz jederzeit für etwas Höheres aufzugeben:

Wenn man aber dafür, dass eine politische Revolution, eine Liebesbegegnung, eine Erfindung der Wissenschaft oder eine Schöpfung der Kunst als verschiedene Unendlichkeiten denkbar werden, die Immanenz (was ich nicht glaube, aber das steht auf einem anderen Blatt) [...] letztlich opfern muss, so werde ich es tun. ${ }^{39}$

Was für Badiou offensichtlich mehr zählt als die Immanenz, ist die Unendlichkeit. So schreibt er auch in Bezug auf die Liebe, sie sei »eine Erklärung der Ewigkeit«, ein »Hinabsteigen der Ewigkeit in die Zeit«. Erst in diesem vertikalen Bezug wird für Badiou die Intensität der Liebe erklärbar: »Deshalb ist sie ein so intensives Gefühl. $\aleph^{40}$ Was das Ereignis verspricht, ist daher nichts Geringeres als eine »Aufhebung der Zeit «, ${ }^{41}$ welche dem einer Wahrheit dienenden Subjekt »im Bruch mit der unerbittlichen Gewöhnlichkeit der Zeit" und »jenseits der Überlebensnotwendigkeit des Menschentiers" erlaube, "unsterblich zu werden ${ }^{42}{ }^{42}$

Die ereignishafte Intensität wird von Badiou also nicht durch eine horizontale Leistung der Immanenz erklärt, sondern durch den vertikalen Transzendenzoperator der Unendlichkeit. Wie er selbst zugibt, übernimmt Badiou hier die christliche Idee, »dass es in der scheinbaren Kontingenz der Liebe ein Element gibt, das nicht auf diese Kontingenz reduzierbar ist «. Einmal mehr behauptet er zwar, dass er - im Gegensatz zum Christentum - dieses »universelle Element [...] als immanent « verstehe. ${ }^{43}$ Trotzdem setzt er genau an dieser Stelle den entscheidenden Schnitt zwischen der kontingenten Ordnung der Immanenz und jener anderen Ordnung der Gnade, in welcher das Ereignis sich vollzieht:

37 Dath: Lost (Anm. 7), 15.

38 Badiou: Lob der Liebe (Anm. 31), $32 \mathrm{f}$.

39 Alain Badiou: Deleuze. »Das Geschrei des Seins«. Übers. v. Gernot Kamecke. Zürich/Berlin 2003, 129f.

40 Badiou: Lob der Liebe (Anm. 31), 45.

41 Badiou: Deleuze (Anm. 39), 93.

42 Badiou: Paulus (Anm. 2), 84.

43 Badiou: Lob der Liebe (Anm. 31), 56. 
»Daraus, dass eine Ereignisstätte existiert, folgt in keiner Weise das Auftreten des Ereignisses. Dieses Auftreten setzt zwar die Immanenz voraus, gehört aber nichtsdestoweniger der Ordnung der Gnade an.«Diese »ereignishafte Gnade« ist durch ihre "Grundlosigkeit» gekennzeichnet. Sie vollzieht sich in einem »Regime des Ohne Ursache« und geschieht »absolut grundlos «. ${ }^{44}$

Die Gnade des Ereignisses erscheint zwar in der kontingenten Singularität einer Stätte, gehört in ihrer absoluten Grundlosigkeit jedoch einer anderen Logik als jener des säkularen Denkens an, welche jedes Geschehen in einem immanenten Netz kontingenter Ursachen verortet. Man wird deshalb weder Badious Rede von einem "Materialismus der Gnade« noch seiner Bestimmung des Ereignisses als »illegale[r] Kontingenz« folgen können. ${ }^{45}$ Das Ereignis ist tatsächlich nicht von dieser Welt, es operiert von einem nicht positiv bestimmbaren Ort jenseits der Immanenz aus und kennzeichnet Badious Theorie damit als eine weitere Variante jener negativen Theologie, die seit Heidegger das ontologische Denken bestimmt. ${ }^{46}$

\section{Jenseits der Verlorenheit: Lost, der Universalismus und die messianische Konstante}

Lost praktiziert dasselbe Verwirrspiel: Die Serie signalisiert immer wieder den guten Willen zu immanenten Erklärungen und kontingenten Herleitungen, etwa wenn sie die Präsenz von Eisbären in den Tropen durch die Experimente der Dharma Initiative plausibilisiert, den Physiker Daniel Faraday als wissenschaftliche Anlaufstelle für die Mysterien der Insel aufbietet oder den metaphysischen Konflikt zwischen Jacob und dem Man in Black auf die nur allzu menschliche Dynamik einer manipulativen Mutter zurückführt. Trotzdem endet das Ganze schließlich doppelt religiös: einerseits was die Insel betrifft in der heiligen Höhle mit der Bewahrung des universalen Lebenslichts und andererseits was die Figuren betrifft in der Kirche über der Lamp Post Station mit der konfessionsunabhängigen Auferstehung in die gleißende Helle der Erlösung. Der Dualismus von Lost zeigt sich zudem besonders stark in der formalen

44 Badiou: Paulus (Anm. 2), 89, 98. Statt von Gnade spricht Badiou manchmal auch vom "Mysterium des Überschusses" und postuliert die Existenz geheimnisvoller »unbenennbarer bzw. ununterscheidbarer Teile«, sodass am Ende gerade "dasjenige, das sich nicht unterscheiden lässt, in Wirklichkeit die allgemeine Wahrheit einer Situation ist, die Wahrheit ihres eigentlichen Seins«. Ders.: Das Sein und das Ereignis (Anm. 26), 319, 543, 369.

45 Badiou: Paulus (Anm. 2), 118, 102.

46 Vgl. dazu Marchart: Die politische Differenz (Anm. 27), zu Badiou insbesondere 152-177, ebenso Daniel Strassberg: Der Wahnsinn der Philosophie. Verrückte Vernunft von Platon bis Deleuze. Zürich 2014, 339. 
Spaltung der letzten Staffel. Während eine Erzählebene die Rettung der insularen Singularität behandelt, transzendieren die Seitwärtsblenden das Inselnarrativ auf seine universale Bedeutung hin. Die Parallelführung dieser beiden Ebenen während der gesamten sechsten Staffel verknüpft formal die singulären Geschehnisse auf der Insel mit der Universalität der Gnade, welche den Protagonisten in der Seitwärtswelt widerfährt.

In den letzten Minuten der finalen Folge schließlich wird enthüllt, dass diese universale Ebene nicht in einer parallelen Sideways-Realität spielt, sondern in Wahrheit Vorblenden in das Leben nach dem Tod erzählt, wo die Figuren erneut verloren umherirren, ohne Erinnerung an alles, was auf der Insel geschah. Während die Vorblenden der dritten und vierten Staffel jedoch die unvollständige Erlösung der bloß physischen Rettung zeigten, komplettieren die JenseitsVorblenden der sechsten Staffel nun die Erlösung der Charaktere, indem diese auch noch aus ihrer metaphorischen Verlorenheit errettet werden. Das messianische Ereignis vollzieht sich also auch hier als Operation der Unendlichkeit, es lässt die Ewigkeit in die Zeit hinabsteigen und seine Subjekte unsterblich werden. Dies allerdings kann nur im Futur II erzählt werden. So wie für Badiou die Sprache eines Subjekts, insofern sie sich auf den Vektor einer erst noch als gültig zu ermittelnden Wahrheit bezieht, nur im Futur II stehen kann, ${ }^{47}$ erzählt auch Lost ein durch die Zukunft zu verifizierendes Ereignis der Gegenwart. So ermitteln die Vorblenden der dritten und vierten Staffel das Scheitern einer rein physischen Rettung: Das bloße Entkommen von der Insel wird kein Ereignis der Erlösung gewesen sein. Die Vorblenden der sechsten Staffel hingegen bestätigen nun die Gültigkeit der Gnade: Erst im Erkennen der Wahrheit wird sich die Erlösung ereignet haben.

Doch wie zeigt sich die Wahrheit in Lost? Sie erscheint wie in Prousts Auf der Suche nach der verlorenen Zeit als unfreiwillige Erinnerung, die von einem singulären Triggerpunkt ausgelöst wird. ${ }^{48}$ Im Futur II der Jenseits-Vorblenden ist Desmond der Erste, der die Wahrheit erkennt. Sie begegnet ihm in Form eines farbgesättigten, symphonisch untermalten Intensivbilderstroms - allerdings nicht vom Geschmack einer in Tee getunkten Madeleine hervorgerufen, sondern durch die Bestrahlung eines Magnetresonanztomographen. Dem Magnetfeld der Kernspinresonanz ausgesetzt wie einst den Strahlungsemissionen der Swan

47 Badiou: Das Sein und das Ereignis (Anm. 26), 551. Vgl. dazu auch 444-458.

48 Vgl. Marcel Proust: Auf der Suche nach der verlorenen Zeit. Übers. v. Eva Rechel-Mertens u. Luzius Keller. Frankfurt a. M. 2004, v. a. Bd. 1: Unterwegs zu Swann, 66-71, u. Bd. 7: Die wiedergefundene Zeit, 257-333. Im Unterschied zu Lost und Badiou verzichtet Proust allerdings auf einen theologischen Überbau und lässt seine Wahrheiten stattdessen in den immanenten Intensitätskoordinaten einer singulär-kontingenten Erlebnisperspektive kreisen. Dadurch kann er jenen Autoritarismus der Transzendenz vermeiden, dem - wie sich zeigen wird - letztlich sowohl Lost als auch Badiou verfallen. 
Station, hat Desmond plötzlich eine Vision: Er sieht eine hastig notierte Botschaft mit dem Namen »PENNY « (6.11) - ein Erinnerungsfetzen aus der Looking Glass Station, wo Charlie einmal eine kurze Funkverbindung zu Desmonds großer Liebe Penny hatte herstellen können (3.23). Der Soundtrack von Michael Giacchino tupft die ersten sachten Klaviertöne aus Desmonds Leitmotiv über die Szene, dann lässt er die Streicher anschwellen, während eine schnell geschnittene Sequenz mit Bildern aus dem gemeinsamen Leben von Desmond und Penny vorbeirauscht: die erste Begegnung vor dem Kloster, aus dem Desmond eben hinausgeworfen wurde, Küsse, die Geburt ihres Kindes und die Wiedervereinigung, nachdem Desmond von der Insel entkommen ist, schließlich Pennys Stimme: "Desmond? « (6.11) Was ihm hier widerfährt, wird im Lauf der sechsten Staffel mit verschiedenen Triggerpunkten und individuell bebilderten Ereignissequenzen einer ganzen Reihe von Figuren zuteil: Hurley (6.12), Ben (6.16), Sun und Jin, Shannon und Sayid, Kate, Claire und Charlie, Locke, Juliet und Sawyer und ganz zuletzt auch Jack (6.17/18). Aber um zu verstehen, was sich in diesen Sequenzen genau zeigt, muss man mindestens zwei Staffeln zurückspulen.

Einem Vorschlag Daths folgend, kann man die Folge The Constant (4.5) als "Hebelpunkt « für die Lost-Exegese insgesamt betrachten, als eine Art »Gipfel der Selbsterleuchtung des Werks «. ${ }^{49}$ In der Tat erzählt diese um Desmond kreisende Episode bereits exemplarisch, wie Lost seine Figuren in der sechsten Staffel schließlich aus ihrer Verlorenheit zu erlösen gedenkt. Dazu muss die lostness jedoch noch einmal radikalisiert und ebenfalls ins Exemplarische überhöht werden: Zum Frachter hinausfliegend gerät der Hubschrauber mit Desmond an Bord in ein Unwetter und überquert den elektromagnetischen Ereignishorizont der Insel daher nicht exakt an der vorgesehenen, von Faraday als sicher berechneten Übergangsstelle - wodurch Desmonds Bewusstsein im Raum-ZeitGefüge verloren geht und unkontrolliert zwischen 1996 und 2004 herumspringt. Auf dem Frachter liegt bereits ein aus der Nase blutender Funker in der Krankenstation, dem offenbar dasselbe passiert ist und dessen Gehirn bald schon aus Überforderung kollabiert. Damit stellt die Erzählung klar, dass sie Verlorenheit an sich - lostness in Reinform - als tödlich begreift: Das Verfallen an die horizontale Willkür der Partikularzeit ist ein Verhängnis von potenziell fatalem Ausmaß.

Was also ist zu tun? Gibt es eine Rettung aus der absoluten Verlorenheit? Der Faraday des Jahres 2004 schickt Desmond nach Oxford, wo die 1996er-Version von Faraday forscht und das lostness-Phänomen sogleich an seiner Laborratte Eloise testet. Das Tier stirbt und Faraday begreift: "I think Eloise's brain shortcircuited. The jumps between the present and the future, she eventually ... she

49 Dath: Lost (Anm. 7), 68, 65. 
couldn't tell which was which. She had no anchor.« Desmond blickt ihn verständnislos an: "What do you mean - anchor?« Und jetzt, vor seiner mit Gleichungen vollgekritzelten, das ganze Bild ausfüllenden Wandtafel in Richtung eines unsichtbaren Zusammenhangs gestikulierend, setzt Faraday zur großen Erklärung an:

Something familiar in both times. All this, see this? This is all variables. It's random, it's chaotic. Every equation needs stability, something known. It's called a constant. Desmond, you have no constant. When you go to the future nothing there is familiar. So if you want to stop this, then you need to find something there, something that you really, really care about that also exists back here in 1996.

Desmond, der große Romantiker im Ensemble der Verlorenen, hat nur einen Gedanken: "This constant, can it be a person?« (4.5) Zurück im Jahr 2004 versucht Desmond vom Frachter aus eine telefonische Verbindung nach London herzustellen, um jene Penny anzurufen, die er acht Jahre zuvor verlassen hatte, weil er sich selbst erst beweisen musste, dass er ihrer Liebe würdig sei. So verschlug es ihn zur Armee und danach auf eine Segeltour rund um die Welt, von dort auf die Insel zum 108-Minuten-Computer in der Swan Station und nun auf diesen Frachter, an ein rotes Telefon, das ihn entweder retten oder vernichten wird.

Auf der Tonspur erklingen wieder die Klavierakkorde von Desmonds Thema, während aus seiner Nase bereits Blut rinnt. Ein Klicken in der Leitung und jetzt heben die Streicher an, als Penny sich meldet und ihn mit jenem erlösenden »Desmond?« anspricht, das dieser zwei Staffeln später im Tomographen noch einmal hören wird. Die Szene spielt passenderweise an Heiligabend, dem Geburtsfest des Messias, und als das Bild zu Penny wechselt, erscheint sie deshalb vor dem religiösen Glanz eines erleuchteten Weihnachtsbaums. Das Leitmotiv schraubt sich in die Höhe und entfaltet seine volle melodische Pracht, während die Tränen fließen und das Ferngespräch sich durch die Störgeräusche hindurch zum verschlungen Finale immer schnellerer Wechsel zwischen den beiden Gesichtern und Stimmen intensiviert: "»I don't know where I am but ... $>>$ I'll find

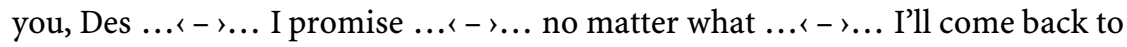

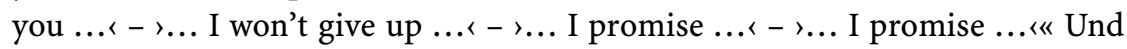
dann gleichzeitig im Chor, bevor totes Rauschen die Verbindung kappt: "»I love you. « Damit ist Desmond vorerst gerettet und die Episode zu Ende, abgesehen von einem Epilog, der die Universalität der gerade präsentierten Heilmethode unterstreicht. Der Faraday des Jahres 2004 sitzt am Strand und blättert in seinem alten Notizbuch, bis er schließlich auf jenen Vorsatz stößt, mit dem er sich später in der fünften Staffel retten wird, als die Insel selbst zwischen den Zeiten zu oszillieren beginnt und das Nasenbluten epidemisch zu werden droht: "IF 
ANYTHING GOES WRONG, DESMOND HUME WILL BE MY CONSTANT.« (4.5)

Was Lost als Erlösung erzählt, ist die Konstruktion eines Fixpunkts im zufälligen Chaos der Welt, die Verankerung eines universalen Eichwerts, der in der Variabilität der Zeiten und Orte immer derselbe bleibt: Wer seine Existenz nicht über eine derart universale Konstante stabilisiert, wird sich in der Partikularität des Seins immer wieder verlieren; wer jedoch eine Konstante fixiert, ist gerettet. Badiou vertritt eine ähnliche Theorie der Subjektivierung, wenn er die Konsistenz des Subjekts von der Treue zu einem Ereignis abhängig macht. Deshalb muss der Zufall des Ereignisses umgehend »fixiert " oder sogar »besiegt« werden. Die Treue fixiert das Ereignis so, dass es »rückwirkend überhaupt nicht mehr als kontingent und zufällig erscheint, sondern praktisch als eine Notwendigkeit«. Das Heil wird auch hier in der Stabilität gesucht, man rettet sich aus der Verlorenheit der Kontingenz in die starken Arme des Schicksals. Denn die Treue arbeitet am »Übergang vom Zufall zum Schicksal«, sie versucht, die flüchtige Zufälligkeit im Beton einer »Konstruktion« zu fixieren, »die so fest ist, als wäre sie notwendig gewesen«. Kontingenz stellt hier also immer ein Risiko dar, das überwunden werden muss: Es braucht zwar den Zufall einer Begegnung, aber die allgemeine Flüchtigkeit ist so groß, dass man auf der Stelle Maßnahmen treffen muss, »damit die Begegnung von ihrem Zufall befreit wird«. So verstanden, ist Treue nichts anderes als ein fortwährender Krieg gegen die Kontingenz, eine stetige Aussortierung des Zufalls aus der Schicksalhaftigkeit des Ereignisses. Ihr Ziel liegt in der Konstruktion einer Konstante als dem »in der Geburt einer Welt besiegten Zufall der Begegnung ${ }^{50}{ }^{50}$ Je totaler der Sieg, desto stabiler die Konstante.

Die Stabilität einer Konstante ist daher abhängig von jenen Punkten, welche das Subjekt zwingen, sich noch einmal zu entscheiden: "Ein Punkt ist ein besonderer Moment, auf den sich ein Ereignis zusammenzieht, wo es in gewisser Weise von neuem gespielt werden muss, so als ob es in einer verschobenen, veränderten Form zurückkäme «. ${ }^{51}$ Entscheidet sich das Subjekt gegen die Treue, zerfällt die Wahrheit des Ereignisses und damit auch das Subjekt selbst. Es kann nur existieren, "insofern es bestätigt, dass es eine gewisse Zahl von Punkten festhalten kann «. ${ }^{52}$ Der Messianismus der Konstanz beruht daher auf dem »Imperativ [...], nichts zu Bruch gehen $\mathrm{zu}$ lassen ${ }^{53}$. Und er veranlasst eine

50 Badiou: Lob der Liebe (Anm. 31), 42ff. Insofern praktiziert Badiou hier genau das, was er der transzendenten oder metaphysischen Orientierung des Denkens vorwirft: Er »sucht einen Haltepunkt für das Umherirren festzulegen«. Ders.: Das Sein und das Ereignis (Anm. 26), 319.

51 Badiou: Lob der Liebe (Anm. 31), 46.

52 Badiou: Logiken der Welten (Anm. 28), 69.

53 Badiou: Paulus (Anm. 2), 69. 
entsprechende Prüfungsmoral: Es genügt nie, das Ereignis nur einmal zu spielen. Vielmehr wird es immer wieder nötig sein, die Treue eines Subjekts von Neuem zu testen, um die Stabilität der Konstante zu gewährleisten.

So lässt sich das Spiel des Ereignisses in Lost an einer Vielzahl von Punkten beobachten. Die erste Komponente, das Ereignis an sich, ist als solches zu flüchtig, um exakt bestimmt zu werden. Aber allgemein kann man sagen, dass es mit dem Zusammenfinden und dem gegenseitigen Sich-wichtig-Werden jener Menschen zu tun hat, welche sich an der Ereignisstätte der Insel begegnen: Das Ereignis lässt diese Figuren füreinander zu Konstanten werden und rettet sie dadurch aus ihrer Verlorenheit. Weil das Ereignis jedoch, wie Badiou sagt, "sich nur jenseits der Situation anzeigen « kann, in der es "aufgetreten ist $",{ }^{54}$ kann es nicht bereits auf der Insel sichtbar werden. Man wird deshalb bis zu den JenseitsVorblenden der sechsten Staffel warten müssen, in denen schließlich die Konstruktion jener Welt sichtbar wird, welche mit den Worten Daths »ein anderes Kontinuum [...], ein rein soziales « herstellt. ${ }^{55}$ Im Serienfinale wird dieses rein soziale Kontinuum von Jacks Vater als das enthüllt, was es ist: "This is a place that you all made together, so that you could find one another." (6.18)

Die zweite Komponente besteht in der Treueprozedur, die das Ereignis bewahrheitet. Sie findet an mehreren Punkten statt. Man kann etwa die Rückblenden der ersten Staffeln mit all ihren überzähligen Begegnungen als jene Betonierung der Notwendigkeit verstehen, welche dem Zufall des Zusammenfindens rückwirkend die Stabilität des Schicksals aufprägt. Wenn andererseits die Oceanic Six in den Vorblenden der vierten Staffel ihre Erlebnisse auf der Insel verschweigen und durch Lügen maskieren, so lässt sich dieser Punkt als ein Scheitern der Treueprozedur bestimmen, sodass jenes Phänomen erscheint, das Badiou Verrat nennt: die Leugnung des Ereignisses. ${ }^{56}$ Demgegenüber können Jacks und Hurleys Übernahme des Inselpatronats am Ende der sechsten Staffel als Punkte der Treue gelten, an denen die Wahrheit des Ereignisses zum Erscheinen gebracht wird.

Die dritte Komponente, die Subjektivierung, wird vor allem in den Vorblenden der sechsten Staffel erzählt. An diesem Punkt agieren die Figuren in einer neuen, deutlich weniger verlorenen Subjektivität. Sie tragen gewissermaßen die singulären Spuren des Ereignisses, haben in ihrem Tod jedoch dessen universale Wahrheit verloren und die Insel mit allem, was auf ihr geschehen ist, vergessen. Hier vertritt Lost noch einmal die Badiou'sche These, dass es in der Frage der Subjektivierung weniger um konkrete Moralität, das heißt um be-

54 Badiou: Das Sein und das Ereignis (Anm. 26), 226.

55 Dath: Lost (Anm. 7), 84.

56 Alain Badiou: Ethik. Versuch über das Bewusstsein des Bösen. Übers. v. Jürgen Brankel. Wien 2003, 103. 
stimmte Verhaltensweisen in kontingent-partikularen Situationen geht, sondern um die Offenbarung einer universalen Wahrheit, deren Gnade sich nur im Bekenntnis einlöst. Diese Wahrheit haben die Figuren nicht festgehalten, deshalb muss das Ereignis noch einmal gespielt, noch einmal neu inkorporiert werden. Um sich als Subjekte zu konstituieren, müssen die Figuren deshalb post mortem noch einmal vom Ereignis gefunden werden - und es festhalten: So wie Desmond in seinem Kernspin-Sarg vom Bilderstrom der Ereignisintensität noch einmal zum Leben erweckt wird und die treu wartende Penelope erneut als einzige Konstante seiner Odyssee fixiert. Mit den Worten Badious: "Diese Destination, die ein Subjekt in einer anderen Logik seiner Erscheinung-in-Wahrheit reaktiviert, nennen wir Auferstehung. ${ }^{57}$

Dergestalt reaktiviert, versammeln sich die Geretteten zum großen Finale in der Kirche, wo Jack als Letzter ebenfalls noch erweckt wird. Jetzt versteht man, warum Michael und Ben nicht dabei sein können: Sie werden von Lost nicht als Erlösungssubjekte qualifiziert, weil sie ihren Konstanten nicht treu waren. Michaels Konstante wäre sein Sohn Walt gewesen, aber ihre Beziehung wird von Schuld und Scham zersetzt, nachdem Michael zum Mörder und Verräter wurde, um Walt aus den Fängen der Others zu befreien (2.20, 4.8). Bens Konstante wäre seine Adoptivtochter Alex gewesen, doch am Punkt der Entscheidung verleugnet er sie und setzt ihr Leben aufs Spiel, um sich selbst zu retten (4.9). Jacks Treue hingegen ist universaler als die aller anderen: Während die meisten Figuren bloß eine Begegnung als Konstante festhalten - Desmond etwa fixiert nur Penny -, zeigt Jacks Ereignissequenz eine Vielzahl von Personen und Vorfällen auf der Insel. Obwohl Kate besonders oft darin vorkommt, scheint Jacks Konstante dennoch nicht in einer einzelnen Liebe aufzugehen, sondern sich vielmehr auf die Gemeinschaft der Überlebenden insgesamt zu beziehen, auf das Ereignis ihrer Zeit zusammen. Wie Jacks Vater sagt: »The most important part of your life was the time that you spent with these people.« (6.18) Diese Wahrheit galt es noch einmal zu erkennen, um die Figuren endgültig aus ihrer Verlorenheit zu erlösen und in die Unsterblichkeit auferstehen zu lassen. Jetzt kann Jacks Vater, der auf den nur allzu deutlich sprechenden Namen Christian Shephard hört, die Kirchentüren öffnen und die Protagonisten aus dem Erzählspiel entlassen. Der Endgegner im Lost-Spiel war der Tod, doch man besiegt ihn auf dieselbe Weise, wie man auch der Verlorenheit einer einsamen Insel entkommt - zusammen, sich gegenseitig als Konstante dienend. Das heißt, mit Dath gegen Sartre: »Der Ausweg, das sind die anderen. ${ }^{58}$

57 Badiou: Logiken der Welten (Anm. 28), 84.

58 Dath: Lost (Anm. 7), 86. Zu Lost und Sartre vgl. Deborah Davidson u. Wayne Jebian: Lost Children: Pregnancy, Parenthood, and Potential. In: Laist (Hg.): Looking for Lost (Anm. 6), hier $178 \mathrm{ff}$. 
Dieser Ausweg, so die gemeinsame These von Lost und Badiou, sei zugleich singulär und universal. Er ermöglicht einerseits die singuläre Rettung bestimmter Individuen, beinhaltet andererseits aber auch das universale Element einer umfassenden Erlösung der Menschheit vom Egoismus. Badiou versteht die Liebe dementsprechend als »Gegenbeweis" zur egoistischen »Überzeugung, dass jeder nur seine eigenen Interessen verfolgt«: »Die Liebe ist aber wie jedes Wahrheitsverfahren wesentlich uneigennützig «. Daher hat sie »eine universelle Tragweite " und stellt eine "persönliche Erfahrung der möglichen Universalität" dar. ${ }^{59}$ Ebenso überhöht Lost die Rettung seiner Figuren zu einem mythischen Duell zwischen Egoismus und Uneigennützigkeit. In einem oft zitierten Dialog am Ende der fünften Staffel vertritt der Man in Black die Ansicht, dass Menschen grundsätzlich in der Matrix eines destruktiven Egoismus agieren:»They come. They fight. They destroy. They corrupt. It always ends the same." Jacob hingegen beruft sich auf einen messianischen Universalismus, der sich im Gegenbeweis einer fundamentalen Uneigennützigkeit der Menschen vollenden werde: »It only ends once. Anything that happens before that is just progress." (5.16)

Doch inwiefern kann ein Ereignis sowohl an der Logik des Singulären wie auch an derjenigen des Universalen teilhaben? Die Logik des Singulären hält fest: Wenn Oceanic 815 durch einen von Desmond verursachten elektromagnetischen Unfall über der Insel abgestürzt ist, dann ist auch die aufgrund dieses Absturzes entstehende Gemeinschaft der Verlorenen ein kontingent-partikulares Geschehen, welches seine lokale Bedeutung für die konkrete Situation und die davon betroffenen Menschen nicht übersteigt. Die Logik des Universalen wiederum behauptet: Wenn diese Leute als Teil von Jacobs Wette gegen den Man in Black auf die Insel gekommen sind, dann ist ihr Zusammenfinden ein Ereignis von globaler Bedeutung, insofern sich darin die Menschheit insgesamt als eine grundsätzlich vom Egoismus erlösbare Spezies erweist. Statt sich nun für eine dieser beiden Varianten zu entscheiden, bestätigt die Serie beide in ihrer jeweiligen Logik und verschweißt diese zwei Ebenen zu dem, was Badiou eine universale Singularität nennt: Das Ereignis, von dem Lost erzählt, erscheint zwar innerhalb der singulären Konstellation eines Unfalls, allerdings nicht als kontingente Folge solcher Partikulareffekte, sondern im Gegenteil als absolut grundlose Gnade, als unvermitteltes Aufblitzen einer universalen Qualität. Wie diese beiden Logiken jedoch ineinander passen sollen - wie und warum genau die Rettung einiger weniger Menschen die Erlösung der gesamten Menschheit beinhalten soll -, bleibt ungeklärt. An diesem Punkt stellt die Serie ihr scheinbar endloses Rätseln ein, um stattdessen zu einem Sprung des Glaubens anzusetzen.

Analog kann es auch in Badious Theorie keinen "Imperativ des Ereignisses" geben ohne den Sprung in jene grundlose Evidenz der Ergriffenheit, welche sich

59 Badiou: Lob der Liebe (Anm. 31), 24, 61. 
ohne weitere Fragen zur Treue verpflichtet fühlt. ${ }^{60}$ Das Ereignis unterliegt keinerlei Deliberation, sondern wird nur in der unmittelbaren Verpflichtung greifbar, es liegt erst dann vor, wenn "etwas geschehen ist, das mich verpflichtet $«{ }^{61}$ Über den Begriff der Gnade hat Badiou das Ereignis zudem gegen jede Nachfrage immunisiert, insofern es seine universale Legitimation gerade aus der Grundlosigkeit bezieht: "Nur was absolut grundlos ist, kann an alle gerichtet sein. « ${ }^{62}$ Man kann einer Grundlosigkeit jedoch schlecht auf den Grund gehen. Was immer sich deshalb über Grundlosigkeit zu legitimieren versucht, verunmöglicht damit a priori jede Debatte über diese Legitimität und fordert stattdessen blinden Gehorsam, unkritische Treue, einen Sprung in die Unmündigkeit des Glaubens. Man sollte sich deshalb weder von Badious Emphase der Entscheidung noch von dem in Lost endlos verhandelten Thema des freien Willens täuschen lassen. Die Entscheidung ist hier immer bereits kuratiert und der freie Wille längst so weit manipuliert, dass man nur noch dem Ruf der Notwendigkeit folgen kann. Denn gemäß Badiou generiert bereits die bloße Existenz eines Ereignisses die Notwendigkeit der Treue: "so gilt nichtsdestotrotz, dass [...] bestimmte Theorie-Ereignisse existieren und dass folglich auch die darauf beruhende Notwendigkeit existiert, diesen treu zu sein. ${ }^{63}$ Hier zeigt sich die Logik des Heiligen, welche ein Phänomen allein schon aufgrund seiner relativen Seltenheit sogleich jeglicher Verhandlung mit dem Profanen entzieht, um ihm stattdessen ein absolutes Privileg zu gewähren: "Zu sagen, alles sei Gnade, bedeutet [...], dass keine Gnade uns jemals erteilt werden kann. Und das ist falsch. Das Sein kommt aus der Unterbrechung zu uns, aus der Ergänzung, und dass es selten ist und zerrinnt, gebietet uns, ihm lange Zeit treu zu bleiben. ${ }^{64}$

Man hat es hier deshalb weniger mit einer Analyse als mit einer Apologie manipulativer Autorität zu tun. Im Fall von Lost zeigt sich das etwa in jenem Gespräch am Lagerfeuer, als Jacob zum letzten Mal auftritt, um einen der verbleibenden Kandidaten zu seinem Nachfolger zu küren. Die Zeit für Erklärungen wird von Jacob sogleich limitiert: "I'll tell you everything you need to know about protecting this island, because by the time that fire burns out, one of you is gonna have to start doing it.« Sawyer misstraut der manipulativen Rede und geht in die Offensive: "What made you think you can mess with my life? I was doin' just fine till you dragged my ass to this damn rock." Nun ist bemerkenswert, wie Jacob auf die Frage Sawyers überhaupt nicht eingeht, sondern bloß dem zweiten Satz widerspricht: »No, you weren't. None of you were. I didn't pluck any of you

60 Badiou: Paulus (Anm. 2), 48.

61 Badiou: Lob der Liebe (Anm. 31), 42.

62 Badiou: Paulus (Anm. 2), 98. In einem ähnlichen Mystizismus operiert der Begriff des Ununterscheidbaren, vgl. oben, Anm. 44.

63 Badiou: Das Sein und das Ereignis (Anm. 26), 273.

64 Badiou: Deleuze (Anm. 39), 134. 
out of a happy existence. You were all flawed.« Dazu erhebt er sich und doziert aufrecht stehend vor den sitzenden Kandidaten, während die Tonspur das zuckersüße There's No Place Like Home-Thema einspielt: »I chose you because you were like me. You were all alone. You were all looking for something that you couldn't find out there. I chose you because you needed this place as much as it needed you.« (6.16)

Mit der Musikwahl stellt sich die Inszenierung klar hinter Jacobs paternalistisches Argument: Verlorene Seelen dürfen jederzeit entführt und diversen Todesgefahren ausgesetzt werden, weil sie dabei ja vielleicht eine Konstante gewinnen. Auf diese Weise werden partikulare Interessenkonflikte von Lost stets in den Synergieeffekten einer universalen Win-win-Situation aufgelöst: Der messianische Universalismus negiert die Konfliktualität des Partikularen zu einer oberflächlichen Differenzillusion, um in den Tiefen des eigentlichen Seins hingegen jene Gnade der ereignishaften Versöhnung zirkulieren zu lassen, welche die Welt zu einer für alle Beteiligten gleichermaßen vorteilhaften Konstellation kompatibilisiert. Sawyers Einwand ist damit vom Tisch und die Kandidaten haben Jacobs Entscheidungsgewalt über sie akzeptiert. Die Frage ist nur noch: "So ... how you gonna pick?» (6.16)

Jetzt, da die Kandidaten ihm die Autorität der Entscheidung übertragen haben, enthüllt Jacob seinen Trumpf: »I'm not going to pick [...]. I want you to have the one thing that I was never given. A choice." Eine Wahl ist nicht etwas, was man immer schon hat, sondern etwas, was einem allenfalls in einem Akt der Gnade gewährt wird - nachdem man bereits darauf verzichtet hat. Kate fragt nach: "And if none of us chooses it? « Darauf Jacob blitzschnell:»Then this ends very badly.« Die Entscheidung ist immer schon auf eine Weise gerahmt, die nur eine Möglichkeit offenlässt. Denn wo immer eine Welt zu retten ist, springt Jack ein, das weiß Jacob ebenso gut wie das Publikum: »I'll do it. This is why I'm here. This is what I'm supposed to do." Jacob stellt die Fangfrage: »Is that a question, Jack?» Doch die opferwillige Christusfigur verneint. Jack hat mittlerweile gelernt, dass das Schicksal keine Fragen beantwortet, sondern nur die Übernahme des Unvermeidlichen gestattet. Jacob nickt befriedigt: »Good.» (6.16) Wer Fragen stellt, disqualifiziert sich als untreu. Nur die bedingungslos Gehorsamen können in die Dienerschaft des Uneigennützigen eintreten.

Sowohl Lost wie auch Badiou entwerfen letztlich ein Subjekt reiner Servilität. Indem das Subjekt nur durch seine Treue zu einem Konstanzpunkt aus der Verlorenheit gerettet werden kann, konstituiert es sich ausschließlich in einem Dispositiv der Verpflichtung und kann sich nie anders denn als ein unmündig dienendes verstehen: Es existiert nur als Treue. Diese Treue verspricht ihrem Subjekt ein Leben jenseits der Verlorenheit, eine von jeglicher Ambivalenz erlöste Existenz. Denn das Heilssubjekt »findet die lebendige Einheit von Denken und Handeln wieder«, wie Badiou schreibt: »Man kann übereinkommen, ১Heik 
Folgendes zu nennen [...]: dass das Denken vom Tun und von der Macht nicht getrennt zu sein braucht. [...] Das ist es, was ich meinerseits eine Wahrheitsprozedur nenne." Den »Schatz« des Ereignisses inkorporierend, weiß sich das Subjekt zudem »von einer unendlichen Macht durchdrungen«: Es agiert im Dienst einer absoluten, für alle gleichermaßen geltenden Wahrheit und ist daher gerechtfertigt, was immer es auch tut - der Win-win-Universalismus garantiert schließlich, »dass jeder Sieg in Wirklichkeit ein Sieg aller ist«. Doch in diesem Versprechen der belohnten Treue steckt auch die Drohung der Vernichtung, sollte die Treue jemals ins Wanken geraten: „Denn wenn das Gefäß zerbricht und der Schatz, den es enthält, in Rauch aufgeht, dann zerbricht auch er, das Subjekt, der Herold, der anonyme Träger. « ${ }^{65}$

\section{Konklusion: Das Erlösungsportal und der Autoritarismus der Transzendenz}

Die Heilstopographie des Universalismus ist simpel: Im Partikularen geht man verloren - im Universalen wird man gerettet. Erlösung winkt stets nur dort, wo die konkrete Realität des Materiellen auf eine allgemeine Idealität hin transzendiert wird. Was den von Lost und Badiou formulierten messianischen Universalismus auszeichnet, ist jedoch gerade die Vehemenz, mit der diese simplen Heilskoordinaten hier verrätselt werden. Die enorme Motivierungsarbeit, mit welcher die Transzendenz des Universalen in die Probleme des Partikularen und die Stätten des Singulären eingebettet wird, kann nur als Indiz dafür gelten, dass an dieser Stelle versucht wird, zwei sich gegenseitig abstoßende Kräfte miteinander zu verbinden. Was da so felsenfest in der Logik des Materialismus verankert werden soll, ist etwas, das die Materialität flieht und von ihr zurückgestoßen wird: die Logik des Religiösen.

Man hat es hier also mit einer jener Expansionsbewegungen zu tun, welche die Grenzlinie zwischen säkularem und religiösem Denken verwischen, um sich bestimmte, vormals der anderen Seite zugehörige Konzepte anzueignen. Denn Lost und Badiou integrieren eine quasireligiöse Eschatologie des Ereignisses in ihren formal säkularen Diskurs. Sie spalten die Immanenz des Kontingenten mit einem ereignishaften Schnitt, um in dieser Unterbrechung das ganz Andere einer unvermittelten Absolutheit aufblitzen zu lassen: jene aus dem Jenseits der

65 Badiou: Paulus (Anm. 2), 109, 105, 69, 119. Analog funktioniert Heideggers Theorie einer im Gehorsam gegenüber dem Gewissensruf unmittelbar handelnden Entschlossenheit, vgl. ders.: Sein und Zeit (Anm. 10), v. a. 267-301. Vgl. dazu auch Stéphane Boutin: Les héros de l'authenticité. Histoires du salut chez Karl May et Heidegger. In: Strenae. Recherches sur les livres et objets culturel de l'enfance 9 (2015). Online unter: http://strenae.revues.org/1456 (01.07.2015). 
Kausalität eingreifende Gnade, welche die Konfliktualität des Partikularen in einer universalen Kompatibilität versöhnt. Als Portal zwischen dem Säkularen und dem Religiösen agiert dabei die These von der Existenz universaler Singularitäten: Dieses Postulat öffnet an besonderen Stätten der Immanenz eine Art Wurmloch in die Transzendenz - ein zwischen Ontologie und Science Fiction schillerndes Bermuda-Dreieck, in dem man die Aufklärung verschwinden und das Grundlose erscheinen lassen kann. Über diese Brücke wird die Universalität in das Singuläre eingeschleust und die Unendlichkeit im Materialismus installiert, um das Ereignis zu heiligen, seine Wahrheit dem profanen Streit der Meinungen zu entrücken und seine Treuesubjekte zu erlösen. Allerdings kann der messianische Universalismus dieses Erlösungsportal offenbar nur so konfigurieren, dass es zugleich auch jenen dogmatischen Autoritarismus der Transzendenz hindurchschlüpfen lässt, welcher die servile Subjektivität der Unmündigkeit restauriert und die Kontingenz des Partikularen erneut zu einem erlösungsbedürftigen Jammertal bloßer Verlorenheit abwertet. Absolut ist hier deshalb sowohl die Verheißung als auch der Preis der Unterwerfung, den sie verlangt. Doch der Messianismus war von jeher ein Diskurs der Sirenen, deren Verlockung jede Vorsicht in den Wind schlägt. 